\title{
Emergence of KPC-2-Producing ST11-K64 Hypervirulent Klebsiella Pneumoniae for the Elderly in Intensive Care Unit: Antimicrobial Resistance, Molecular and Clinical Characteristics
}

\section{Tian Wei}

Shanghai University of Traditional Chinese Medicine Yueyang Hospital of Integrated Traditional Chinese Medicine and Western Medicine https://orcid.org/0000-0002-2398-0326

\section{Cheng-yun Zou}

Shanghai University of Traditional Chinese Medicine

Jie Qin

Shanghai University of Traditional Chinese Medicine

\section{Jian-min Tao}

Shanghai University of Traditional Chinese Medicine Yueyang Hospital of Integrated Traditional Chinese Medicine and Western Medicine

Li Yan

Shanghai University of Traditional Chinese Medicine Yueyang Hospital of Integrated Traditional Chinese Medicine and Western Medicine

\section{Jiang-jun Wang}

Shanghai University of Traditional Chinese Medicine Yueyang Hospital of Integrated Traditional Chinese Medicine and Western Medicine

\section{Hong Du}

Second Affiliated Hospital of Soochow University

\section{Fang Shen}

Fifth People's Hospital of Shanghai Fudan University

\section{Yan-qin Zhao}

Shanghai University of Traditional Chinese Medicine Yueyang Hospital of Integrated Traditional Chinese Medicine and Western Medicine

\section{Hai-ying Wang ( $\square$ liang19961111@126.com )}

Shanghai University of Traditional Chinese Medicine Yueyang Hospital of Integrated Traditional Chinese Medicine and Western Medicine

\section{Research}

Keywords: Carbapenem-resistant klebsieblla pneumoniae, Hypervirulent, Multilocus sequence typing, Risk factor, The elderly 
Posted Date: August 2nd, 2021

DOI: https://doi.org/10.21203/rs.3.rs-746521/v1

License: (c) (7) This work is licensed under a Creative Commons Attribution 4.0 International License. Read Full License 


\section{Abstract}

Background: In recent years, carbapenem-resistant hypervirulent Klebsiella pneumoniae (CR-hvKP) has been increasingly reported and poses severe therapeutic challenge to global public health, but has not yet been systematically studied in elderly patients. This study aimed to investigate the clinical and molecular characteristics and risk factors of CR-hvKP infections in elderly patients.

Methods: We retrospectively investigated elderly patients with carbapenem-resistant Klebsiella pneumoniae (CRKP) infections in intensive care unit (ICU),between January 2020 and December 2020, the clinical data being collected from medical records, and microbiological data including antimicrobial susceptibility testing, phenotype experiment, carbapenemases production, string test, virulence gene, capsular serotype-specific (cps) genes and multilocus sequence typing, of the CR-hvKP group defined by the presence of some combination of rmpA, rmpA2, iucA, iroB, and peg-344 was compared with those of carbapenem-resistant non-hypervirulent Klebsiella pneumoniae (CR-non-hvKP) strains.

Results: Of 80 CRKP strains, 51(63.8\%) met the definition of CR-hvKP and 7 of them had all five of the virulence genes tested. The main mechanism of resistance to carbapenems found in CR-hvKP strains was the presence of the $b / a_{\mathrm{KPC}-2}$ gene. There was no statistical significance in the resistance rates of antimicrobial agents between the CR-hvKP and CR-non-hvKP subgroups $(p \geq 0.05)$. Compared with the minimum inhibitory concentration (MIC) 50 values of CR-non-hvKP group, those of antimicrobials, including ceftazidime, ceftazidime/avibactam, imipenem/avibactam, tigecycline, levofloxacin, cefoperazone-sulbactam, exhibited higher levels in the CR-hvKP group. Avibactam $(4 \mu \mathrm{g} / \mathrm{mL})$ significantly decreased the MIC90 values more than sixteen-fold than that of ceftazidime and imipenem alone against KPC-2-producing Klebsiella pneumoniae. K64 and ST11 were highly prevalent and strongly associated with CR-hvKP $(P<0.05)$. Cardiovascular disease (odds ratio $[\mathrm{OR}]=11.956)$ and ST11-K64 $(\mathrm{OR}=8.385)$ appeared to be independent variables associated with CR-hvKP infection by multivariate analysis.

Conclusion: The CR-hvKP strains showed higher MIC50 values than CR-non-hvKP strains. KPC-2-producing ST11-K64 CR-hvKP is emerging, which might become new significant "superbugs" and a threat to elderly patients.

\section{Introduction}

Klebsiella pneumoniae (K. pneumoniae), an important community-acquired and nosocomial pathogen, especially in the intensive care unit (ICU), causes widespread infections including pneumonia, bacteremia, urinary tract infection, endophthalmitis, liver abscesses and sometimes even life-threatening septic shock. Two clinical pathotypes: hypervirulent Klebsiella pneumoniae (hvKP) and carbapenem resistant Klebsiella pneumoniae (CRKP) often cause life-threatening infections ${ }^{[1]}$. In the conventional sense, hvKP is seldom resistant to antibiotics, however, because of virulence genes and antibiotic resistance genes transmitted by mobile genetic elements, increasing researches show that carbapenem-resistance hypervirulent klebsilla pnemoniae (CR-hvKP) strains are widely found ${ }^{[2-4]}$. In China, the prevalence of CR-hvKP infections is $0 \%$ $25.8 \%$, with Henan and Shandong being the most serious ${ }^{[5]}$. The emergence of CR-hvKP could lead to the next clinical crisis, has attracted worldwide attention ${ }^{[2]}$. 
During the last decade, the global spread, of CRKP posed a severe challenge to public health, particularly in China. According to the China Antimicrobial Resistance Surveillance System report ${ }^{[6]}$, most CRKP strains were isolated from ICU, and were common in the elderly. The main resistant mechanism for CRKP in China is the production of KPC-2 carbapenemase, and ST11 is the dominant sequence type (ST) ${ }^{[7]}$. A recent genomic analysis showed that ST11 classic CRKP strains can acquire the pLVPK-like plasmid and become CR-hvKP strains $^{[2]}$.

In the past, the definition of hvKP relied on clinical manifestations and phenotypes, which proven to have poor specificity and sensitivity ${ }^{[8]}$. The $r m p A, r m p A 2, i u c A$, iroN and peg-344 genes on virulence plasmids can be used for the identification of hvKP and classic KP(cKP) strains with pinpoint accuracy ${ }^{[8]}$. The emergence of CR-hvKP will be a great challenge for clinician and result in adverse outcome. So far, researches on CR-hvKP in elderly patients remain limited ${ }^{[5]}$. Therefore, a cognitive of the risk factors of infections, microbiological and molecular characteristics in CR-hvKP will provide important insights into the control and treatment for CR-hvKP infections among the elderly patients.

In response to this emerging problem, a retrospective study in elderly patients in a Chinese hospital was conducted, the aim was to advance our cognitive of the clinical characteristics, risk factors, hypervirulence and epidemiology of CR-hvKP infections based on newly recognized biomarkers.

\section{Methods}

\section{Bacterial strains and identification}

We collected clinical CRKP strains from patients over sixty-five years old between January 2020 and December 2020 from ICU in Yueyang Hospital of Integrated Traditional Chinese and Western Medicine, Shanghai University of Traditional Chinese Medicine, and the capacity of the hospital is more than 1200 beds. All the strains were collected consecutively and non-duplicated, only the first strain was collected from the same patient. All the K. pneumoniaeidentification and the drug resistance of imipenem and ertapenem were detected by Vitek 2 system (bioMérieux, France) and further species identification was confirmed with MALDI-TOF MS system (Bruker Daltonics, USA), the drug resistance of meropenem was tested by using a Kirby-Bauer disk diffusion susceptibility test. CRKP was defined as that resistant to either imipenem, meropenem or ertapenem ${ }^{[9]}$ in accordance with the breakpoints of 2020 Clinical and Laboratory Standards Institute (CLSI) guidelines $^{[10]}$. We collected all clinical information for CRKP positive patients from electronic medical records, including basic demographics, underlying diseases, clinical presentations, exposure to hospital care, treatment process, and outcomes.

\section{Antimicrobial Susceptibility Testing}

The Antimicrobial Susceptibility Testing (AST) for the CRKP strains were further performed by the broth microdilution assay (BIO-NONT, China). The antibiotics included ceftazidime, ceftazidime/avibactam, cefepime, aztreonam, piperacillin/tazobactam, imipenem,imipenem/

avibactam, meropenem, amikacin, polymyxin B, tigecycline, levofloxacin, cefoperazone/sulbactam. The AST breakpoints of 2020 CLSI guideline were adopted ${ }^{[10]}$ with exception of tigecycline and polymyxin $B$. The 
breakpoint of tigecycline was based on the US Food and Drug Administration(FDA) standard ${ }^{[11]}$,and the breakpoint of polymyxin B was based on the European Committee on Antimictobial Susceptibiity Testing(EUCAST) standard ${ }^{[12]}$. The breakpoints of imipenem/avibactam and cefoperazone/sulbactam were referred to imipenem and cefoperazone in CLSI, respectively ${ }^{[13-14]}$. The standard strains E. coliATCC 25922 and K. pneumoniae ATCC700603 were used as quality control strains in each experiment.

\section{DNA extraction}

The strains were inoculated into the blood agar plate and incubated overnight at $35^{\circ} \mathrm{C}$. The colonies were suspended in $500 \mathrm{ml}$ sterile distilled water. The suspension was boiled at $100^{\circ} \mathrm{C}$ for 10 minutes, centrifuged at $10000 \mathrm{rmp}$ for 13 minutes. The supernatant was used as a template for DNA reaction. The DNA was stored at $35^{\circ} \mathrm{C}$.

\section{Phenotype experiment and detection of Carbapenemases}

We used phenylboronic acid (PBA)and ethylenediamine tetra-acetic acid (EDTA) ${ }^{[15]}$ companying with imipenem to detect the phenotypes of KPC $\beta$-lactamase and metal $\beta$-lactamase (MBLs), respectively. In both tests, compared with imipenem alone, the diameter of the inhibition zone has increased $>$ or $=5 \mathrm{~mm}$ by PBA or EDTA, which is considered to be a positive combined-disc test result that shows the presence of KPC $\beta$-lactamase or MBL enzyme, respectively. The presence of carbapenemase genes ( $b / a_{\mathrm{KPC}}, b / a_{\mathrm{NDM}}, b / a_{\mathrm{IMP}}$ and $\left.b / a_{\mathrm{OXA}-48}\right)$ were detected by PCR as previously described ${ }^{[16-17]}$. The primers are listed in Supplementary table 1. The PCR amplified products were sequenced (Sangon Biotech, Shanghai) and the results were compared with the BLAST searches in the NCBI Genbank.

\section{Detection of virulence-associated features and capsular serotype-specific (cps)genotyping}

Hypermucoviscosity as a virulence factor was identified by the string test. If a viscous string longer than $5 \mathrm{~mm}$ is generated by stretching a bacterial colony on blood agar plate with a bacteriology inoculation loop, the string test is positive ${ }^{[18]}$. Five virulence genes including $r m p A, r m p A 2, i u c A$, iroB, peg-344 ${ }^{[8,19-20]}$ and eight capsular serotype-specific (cps) genes (K1, K2, K5, K20, K47,K54, K57 and K64) ${ }^{[20-23]}$ were identified by polymerase chain reaction(PCR) as previous described. The primers are listed in Supplementary table 1. The CRKP strain which detected with any one of the five virulence genes was defined as CR-hvKP.

\section{Multilocus Sequence Typing (MLST)}

Seven housekeeping genes ( $g a p A$, $m d h$, phoE, ton $B$, infB, pgi and $r p o B$ ) were performed according to the protocol, which were described in online databases. (https://bigsdb.pasteur.fr/klebsiella/

Primers_used.html). The PCR amplified products were sequenced (Sangon Biotech,China), the results were compared by the use of this website.

\section{Statistical analysis}

IBM SPSS Statistics $\llbracket$ version $19.0 \otimes$ was used for statistical analysis. The $\chi 2$ or Fisher's exact test were used for categorical variables. $P<0.05$ was considered statistically significant. To further identify variables associated 
with CR- hvKP infections, Logistic regression was used. All variables with $P<0.05$ were included in the multivariate analysis.

\section{Results}

\section{CRKP strains and Virulence-associated features}

A total of 80 CRKP strains isolated from 80 patients in ICU were included in the study. The percentages of 80 CRKP strains isolated from respiratory tract, urine, blood, pus and puncture fluid were $72.5 \%, 15.0 \%, 6.3 \%, 5.0 \%$ and $1.3 \%$, respectively. CRKP strains were divided as CR-hvKP and carbapenem-resistant non-hypervirulent Klebsiella pneumoniae (CR-non-hvKP) based on whether they contained virulence genes. The detection of the presence of virulence genes showed that 51 (63.8\%) of 80 CRKP strains were positive, suggesting carrying virulence plasmids, which were designated as CR-hvKP group, while the remaining 29 strains were designated as CR-non-hvKP group.

Among the five virulence-associated genes, rmpA2, iucA, iroN, peg-344 and rmpA harboured by 51 strains (Figure 1), the most common virulence gene was $r m p A 2$, the prevalence was $90.2 \%(46 / 51)$, followed by iucA, iroN, peg-344 and rmpA, the detection rate were $88.2 \%(45 / 51), 27.5 \%(14 / 51), 21.6 \%(11 / 51)$ and $19.6 \%$ (10/51), respectively. Seven (13.7\%) strains had all five of the virulence genes, which indicate that the full length of the virulence plasmid pLVPK could be harbored by these strains. The results of the string test showed that $41.2 \%(21 / 51)$ CR-hvKP strains showed negative results in string test, whereas the results of the 17 CR-nonhvKP strains were positive, accounting for $58.6 \%$ (17/29).

For the eight serotypes closely related to hvKP, 65(81.3\%) strains were positive for K47, K54, or K64 serotypes, additionally, there is no strain with $\mathrm{K} 1, \mathrm{~K} 2, \mathrm{~K} 5, \mathrm{~K} 20$ or $\mathrm{K} 57$ serotypes in the present study. Serotype K64 was dominated serotype of CRKP strains [62 (77.5\%) of 80 strains]. The frequency of K64 type (86.3\% vs. $62.1 \%$, $P=0.013)$ identified in CR-hvKP strains was significantly higher than that of CR-non-hvKP strains. K-non-typable (34.5\% vs. 9.8\%, $P=0.007$ ) was strongly associated with CR-non-hvKP. Only two strains were identified as K47 or K54 (Table 1).

\section{Susceptibility results and Carbapenemase resistance Genes}

The antimicrobial susceptibility profiles of the CR-hvKP and CR-non-hvKP strains were listed in Table2. According to the AST results, all strains were resistant to imipenem and meropenem with exception of one CRhvKP strain being susceptible to memropenem, and showed high level resistance to cephalosporins, monobactam, $\beta$-lactams/ $/$ lactamase inhibitor combinations, fluoroquinolones, but were almost not resistant to ceftazidime/avibactam (2.0\% vs. 3.5\%), imipenem/avibactam (3.9\% vs. $3.5 \%)$, polymyxin B (0.0\% vs. $17.2 \%)$ and tigecycline $(0.0 \%$ vs. $0.0 \%)$. Ceftazidime and imipenem by combining avibactam $(4 \mu \mathrm{g} / \mathrm{mL})$ significantly decreased the MIC90 values more than sixteen-fold than that of ceftazidime and imipenem alone against KPC2-producing Klebsiella pneumoniae. Although we found there were no statistical significance in the resistance rates of antimicrobial agents between the two subgroups $(p \geq 0.05)$, except polymyxin $\mathrm{B}(P=0.002)$. The MIC50 values of the following antibiotics of the CR-hvKP strains increased two-fold compared with those of CR-nonhvKP: ceftazidime (>128 vs. $128 \mu \mathrm{g} / \mathrm{mL}$ ), ceftazidime/avibactam $\varangle 4 / 4$ vs. $2 / 4 \mu \mathrm{g} / \mathrm{mL}$ ), imipenem/avibactam 
(0.5/4 vs. $0.25 / 4 \mu \mathrm{g} / \mathrm{mL})$, tigecycline ( 2 vs. $1 \mu \mathrm{g} / \mathrm{mL})$, levofloxacin $(64 \mathrm{vs} .32 \mu \mathrm{g} / \mathrm{mL})$, cefoperazone/sulbactam (>256/128 vs. $256 / 128 \mu \mathrm{g} / \mathrm{mL})$.

According to phenotypic experiment results and detection of carbapenemase genes, all CR-hvKP and $96.6 \%$ $(28 / 29)$ CR-non-hvKP strains showed only produced bla KPC-2 carbapenemase gene and the presence of KPC lactamas. Only one CR-non-hvKP strains showed the presence of MBL enzyme, harbored bla $a_{\mathrm{MP}-4}$.

\section{MLST genotyping}

MLST analysis revealed that ST11 and ST15 were the most common STs in CRKP strains, and ST198 and ST438 were distributed in one strain of CR-hvKP strain and one strain of CR-non-hvKP strain. The frequency of ST11 type (88.2\% vs. $69.0 \%, P=0.034)$ identified in CR-hvKP strains was significantly higher than that of CRnon-hvKP strains, however, the proportion of ST15(9.8\% vs.27.6\%, $P=0.039)$ strains was lower (Table 1). The strains were divided into several clones by combination of capsular genotypes and STs. We found that ST11K64 CRKP strains ( $86.3 \%$ vs.58.6\%, $P=0.005)$ were strongly associated with CR-hvKP, while ST15- K-non-typable (7.8\%vs. $27.6 \%, P=0.017$ ) strains were correlated with CR-non-hvKP (Table 1).

\section{Clinical factors for CR-hvKP infection of elderly patients}

Demographic and clinical factors of patients with CR-hvKP and CR-non-hvKP infections are summarized in Table 1. The age, gender, antibiotic exposure, hospitalization within the last 90 days, or length of stay from CRKP isolation to outcome (In-hospital mortality or discharge) didn't have significant difference between CRhvKP and CR-non-hvKP ( $P>0.05)$. In addition, there was no statistical difference among most underlying conditions such as pulmonary disease, diabetes mellitus, hypertension, cerebrovascular diseases, malignancy, hypoproteinemia and liver abscess ( $P>0.05)$, except cardiovascular disease (60.8\% vs.17.2\%, $P<0.001)$. Gastric tube was more prevalent in CR-non-hvKP group than in CR-hvKP group ( $96.6 \%$ vs.80.4\%, $P=0.045)$, whereas the other invasive procedures and devices had no significant difference. Most of the outcomes between the two groups ( $P>0.05$ ) had no statistical difference, the frequency of change of initial antibiotics due to clinical worsening was higher in CR-hvKP group than CR-non-hvKP group (58.8\% vs.34.5\%, $P=0.036)$ (Table 1).

\section{Risk factors of CR-hvKP infection for the elderly patients}

Nine variables with $P<0.05$ in univariate analysis were included in the multivariate analysis, including K64, Knon-typable, ST11, ST15, ST11-K64, ST15-K-non-typable, cardiovascular disease, gastric tube, change of initial antibiotics due to clinical worsening. The results showed that ST11-K64(odds ratio [OR] $=8.385 ; P=0.003$ ) and cardiovascular disease (OR=11.956; $\mathrm{P} \otimes 0.001)$ were independent predictors of CR-hvKP infections for elderly patients.(Table 3).

\section{Discussion}

More than 30 years ago, a report first described hvKP in China ${ }^{[24]}$, in recent years, reports on CR-hvKP have increased gradually, which poses a greater challenge to clinical treatment due to its high drug resistance and high virulence ${ }^{[5]}$. So far a limited number of data based on the microbiological and molecular characteristics in 
the elderly can be searched. According to the results of China's census in 2021, elderly patients over 65 years old account for $13.5 \%$ of the total population ${ }^{[25]}$, China has entered an aging society. As is known to all, ICU is a relatively popular setting for CRKP infections in hospitals and due to low immune function and cumulative basic diseases, the elderly patients are more prone to nosocomial infections. In this study, we focused on elderly patients hospitalized in ICU, reported antimicrobial resistance, molecular and clinical characteristics of CR-hvKP, and found the main CR-hvKP clone KPC-2-producing ST11-K64 among elderly patients.

It is worth noting that the main cause of liver abscess is hvKP infection ${ }^{[26-27]}$,however, we found that the main specimen type of both CR-hvKP strains and CR-non-hvKP strains isolated from was sputum, and only $7.8 \%$ (4/51) patients with CR-hvKP infections had symptoms of liver abscess, suggesting that CR-hvKP can also invade other parts of the body ${ }^{[28]}$. The production of carbapenemases is the most common mechanism of drug resistant in CRKP strains ${ }^{[29]}, \mathrm{KPC}-2$ was the most frequently identified among CR-hvKP strains ${ }^{[2]}$. As reported in previous studies ${ }^{[30]}$, the CRKP strains were resistant to most antibiotics with exception of ceftazidimeavibactam, imipenem/ avibactam, polymyxin B and tigecycline, only one CR-non-hvKP strain which harbored blalMP-4 displayed resistance to ceftazidime/avibactam, this is consistent with the fact that avibactam is inactive against MBL producing strains ${ }^{[31]}$. Avibactam, as the most effective $\beta$-lactamase inhibitor [32], significantly increased the activity of ceftazidime and imipenem against KPC-producing K. pneumoniae in vitro, which decreased the MIC90 values of ceftazidime and imipenem more than sixteen-fold. We also identified one KPC-2-producing strain exhibited resistant to ceftazidime/ avibactam, with MIC value $16 \mathrm{ug} / \mathrm{mL}$, it might due to OmpK35/36 defects, blaKPC-2 point mutation and high KPC expression ${ }^{[33-34]}$. We found a phenomenon that deserves special attention, namely, compared with CR-non-hvKP strains, CR-hvKP strains exhibited high level MIC50 values, especially ceftazidime/avibatam, imipenem/avibatam, tigecycline, which are our last line in treating CRKP-infected patients.

Previously, a positive string test was used as screening test for hvKP, the definition of hvKp is controversial. so we employed five virulence genes, including two regulator genes which have been associated with hypermucoviscous phenotype ( $r m p A$ and $r m p A 2)$, siderophore aerobactin (iucA) and salmochelin (iroB) ${ }^{[5]}$ and the putative metabolite transporter peg344 $4^{[35]}$ attribute to the hypervirulent phenotype of $K$. pneumoniae. We found 51 strains which defined as CR-hvKP harbored a part of combination of these markers, rmpA2 and icuA were the most common virulence genes in our study ${ }^{[36]}$. In term of the thick polysaccharide capsules encoded by the $\mathrm{cps}^{[1]}$, unlike carbapenem-sensitive hypervirulent $K$. pneumoniae which belong to serotypes $\mathrm{K} 1, \mathrm{~K} 2$, or $\mathrm{K}_{57}{ }^{[37]}$, the most popular CR-hvKP clone was K64 in our study ${ }^{[38]}$. The most predominant MLST type of the 80 strains was ST11 ${ }^{[39]}$. Notably $86.3 \%(44 / 51)$ CR-hvKP strains belonged to ST11-K64 clone, which was an independent predictor factor for CR-hvKP infections, being more resistant and exhibited elevated virulence, leading to a high mortality and transmissibility in infected patients ${ }^{[5,40-41]}$. Since all the strains were collected from the same ward ICU, we speculated clonal dissemination of KPC-2-producing ST11-K64 K. pneumoniae exited, and during the process of transmission, KPC-2-producing ST11-K64 K. pneumoniae acquire of a PLVKPlike virulence plasmid, leading to KPC-2-producing ST11-K64 CR-hvKP. ZHANG et al ${ }^{[42]}$ also provided evidence that ST11-K64 K.pneuminiae may be a competent host strain for a hypervirulent plasmid. The emergence of KPC-2-producing ST11-K64 CR-hvKP might pose a serious challenge to the management of elderly patients infected with these strains in the future, as these strains contain both drug resistance and virulence genes and show higher resistance than CR-non-hvKP strain s $^{[32]}$. 
In order to take appropriate intervention measures correctly, a better understanding of risk factors is essential. Univariate analysis showed that change of initial antibiotics due to clinical worsening was strongly associated with CR-hvKP. This reminds the individualized therapy must be used to treat CR-hvKP infections based on in vitro antimicrobial susceptibility profiles, molecular type, and the patient's health status ${ }^{[43]}$. Cardiovascular disease was independent predictor to CR-hvKP infections. Patients with underlying diseases are more likely to develop hvKP infections ${ }^{[4]]}$, such patients typically receive inappropriate empiric therapy, more frequent hospital exposure associated with severe comorbidities may result in exposure to CR-hvKP and possible infections ${ }^{[45]}$. Meanwhile, older age and serious complications could reduce immunity and thus increase the risk of infection/settlement and even death ${ }^{[46]}$. Clinicians should pay close attention to these risk factors in clinical practice to reduce emergence of CR-hvKP strains.

There were some limitations in our study. First, this is a one-year retrospective study conducted in a single center, not a multi-center, longitudinal molecular epidemiological study on CR-hvKP. Second, Pulsed field gel electrophoresis was not done in this study, we speculated nosocomial transmission based on MLST typing, capsule serotype and drug resistance genotype of the clinically strains.

\section{Conclusions}

CR-hvKP is emerging as a common pathogen in the elderly in China. Cardiovascular disease and ST11-K64 appeared to be independent variables associated with CR-hvKP infections. We firstly found the CR-hvKP strains showed higher MIC50 values than CR-non-hvKP strains. KPC-2-producing ST11-K64 CR-hvKP might become new significant "superbugs" and a threat to elderly patients.

\section{Abbreviations}

AST: antimicrobials susceptibility testing; BLAST: Basic Local Alignment Search Tool; cKP: classic K. pneumoniae; CLSI: Clinical and LaboratoryStandards Institute;cps:capsular serotype-specific; CRKP: Carbapenem-resistant Klebsiella pneumoniae,CR-hvKP: carbapenem-resistant hypervirulent Klebsiella pneumoniae; CR-non-hvKP:carbapenem-resistant non-hypervirulent Klebsiella pneumoniae; PBA:Phenyloronic acid; EDTA:ethylenediamine tetra-acetic acid; E. coli: Escherichia coli. EUCAST: European Committee on Antimicrobial Susceptibility Testing; FDA:Food and Drug Administration; hvKP: hypervirulent Klebsiella pneumoniae; K. pneumoniae: Klebsiella pneumoniae, ICU: intensive care unit ;KPC: Klebsiella pneumoniae carbapenemase; MBL:metal $\beta$-lactamase; MIC: Minimal Inhibitory Concentration; MLST: Multilocus sequence type; NCBI: National Center for Biotechnology Information; OR: odds ratio: PCR: Polymerase Chain Reaction; PFGE: Pulsed-field gel electrophoresis; SPSS: Statistical Package for the Social Sciences; ST: Sequence Type;

\section{Declarations}

\section{Ethics approval and consent to participate}

Informed consent was not needed due to retrospective nature of the study. The study was approved by the by the research Ethics Committee of Yueyang Hospital of Integrated Traditional Chinese and Western Medicine区 Shanghai University of Traditional Chinese Medicine, Shanghai, China (approval number (2021)060). 


\section{Consent for publication}

Not applicable.

\section{Availability of data and materials}

The datasets used and/or analysed during the current study are available from the corresponding author on reasonable request.

\section{Competing interests}

The authors declare that they have no competing interests.

\section{Funding}

This work was supported by Foundation of Shanghai Municipal Health Commission for Distinguished Young Scholar project (grant number: 20204Y0147); the Excellent Youth incubator fund Foundation of Yueyang Hospital of Integrated Traditional Chinese and Western Medicine, Shanghai University of Traditional Chinese Medicine (grant number 2019YYQ30).

\section{Authors' contributions}

TW and HYW were responsible for study design and article writing. CYZ and JQ performed PCR and statistical analyses. JMT, LY and JJW collected the laboratory and clinical data. TW, HD, FS, YQZ and HYW performed critical data review. All authors read and approved the final version of the manuscript.

\section{Acknowledgements}

We thank the team of curators of the Institut Pasteur MLST and whole genome MLST databases for curating the data and making them publicly available at http://bigsdb.pasteur. fr. Additionally, we thank Xing Wang for suggestions given during the experiment, who works in Shanghai Childen's Mendical Center, Shanghai, China.

\section{Author details}

*Correspondence: liang19961111@126.com

${ }^{1}$ Department of Clinical Laboratory,Yueyang Hospital of Integrated Traditional Chinese and Western Medicine, Shanghai University of Traditional Chinese Medicine, Shanghai, China.

${ }^{2}$ Shanghai University of Traditional Chinese Medicine, Shanghai, China.

${ }^{3}$ Department of Clinical Laboratory, The Second Affiliated Hospital of Soochow University, Suzhou, China.

${ }^{4}$ Department of Clinical Laboratory, Shanghai Fifth People's Hospital, Fudan University, Shanghai, China.

\section{References}


1. Gomez-Simmonds A, Uhlemann AC. Clinical Implications of Genomic Adaptation and Evolution of Carbapenem-Resistant Klebsiella pneumoniae. J. Infect. Dis. 2017;215 Suppl 1: 18-27.

2. Lee CR, Lee JH, Park KS, Jeon JH, Kim YB, Cha CJ, Jeong BC, Lee SH. Klebsiella pneumoniae Antimicrobial Resistance of Hypervirulent: Epidemiology, Hypervirulence-Associated Determinants, and Resistance Mechanisms. Front Cell Infect Mi. 2017;7: 483.

3. Zhan L, Wang S, Guo Y, Jin Y, Duan J, Hao Z, Lv J, Qi X, Hu L, Chen L, Kreiswirth BN, Zhang R, Pan J, Wang L, Yu F. Klebsiella pneumoniaeOutbreak by Hypermucoviscous ST11 Isolates with Carbapenem Resistance in a Tertiary Hospital in China. Front Cell Infect Mi. 2017;7:182.

4. Zhang R, Lin D, Chan EW, Gu D, Chen GX, Chen S. Emergence of Carbapenem-Resistant Serotype K1 Hypervirulent Klebsiella pneumoniae Strains in China. Antimicrob Agents Ch. 2016;60(1):709-11.

5. Zhang Y, Jin L, Ouyang P, Wang Q, Wang R, Wang J, Gao H, Wang X, Wang H. Evolution of Hypervirulence in Carbapenem-Resistant Klebsiella Pneumoniae in China: A Multicentre, Molecular Epidemiological Analysis. J. Antimicrob. Chemother. 2020;75(2):327-36.

6. Expert Committee on Rational Drug Use of the National Health Commission. 2019 National Bacterial Drug Resistance Monitoring Report. China antimicrobial resistance surveillance system.

2019.http://www.carss.cn/Report/ 2019 National Bacterial Drug Resistance Monitoring Report. Accessed 19 Nov 2020.

7. Wang Q, Wang X, Wang J, Ouyang P, Jin C, Wang R, Zhang Y, Jin L, Chen H, Wang Z, Zhang F, Cao B, Xie L, Liao K, Gu B, Yang C, Liu Z, Ma X, Jin L, Zhang X, Man S, Li W, Pei F, Xu X, Jin Y, Ji P, Wang H. Phenotypic and Genotypic Characterization of Carbapenem-Resistant Enterobacteriaceae: Data From a Longitudinal Large-Scale CRE Study in China (2012-2016). Clin Infect Dis. 2018;67 Suppl 2:196-205.

8. Russo TA, Olson R, Fang CT, Stoesser N, Miller M, MacDonald U, Hutson A, Barker JH, La Hoz RM, Johnson JR. Identification of Biomarkers for Differentiation of Hypervirulent Klebsiella Pneumoniae From Classical K. Pneumoniae. J Clin Microbiol. 2018;56(9): e00776-18.

9. Guidance for Control of Infections with Carbapenem-Resistant or Carbapenemase-Producing Enterobacteriaceae in Acute Care Facilities. MMWR. Morbidity and mortality weekly report. 2009;58(10):256-60.

10. Clinical and Laboratory Standards Institute. Performance standards for antimicrobial susceptibility testing, 30th ed CLSI supplement M100. Clinical and laboratory standards institute. Wayne: Clinical and Laboratory Standards Institute; 2020.

11. Antimicrobial Susceptibility Test Interpretation Criteria.tigecycline-injection-products. The Food and Drug Administration.2019.https://www.fda.gov/drugs/development-resources/tigecycline-injection-products. Accessed 17 JUN 2019.

12. The European Committee on Antimicrobial Susceptibility Testing. Breakpoint Tables for Interpretation of MICs and Zone Diameters, version 9.0.2019. http://www.eucast.org/clinical_breakpoints/. Accessed 18 Jan 2019.

13. Aktaş Z, Kayacan C, Oncul O. In Vitro Activity of Avibactam (NXL104) in Combination with B-Lactams Against Gram-negative Bacteria, Including OXA-48 B-Lactamase-Producing Klebsiella Pneumoniae. Int J Antimicrob Ag. 2012;39(1):86-89. 
14. Lan P, Jiang Y, Zhou J, Yu Y. A Global Perspective On the Convergence of Hypervirulence and Carbapenem Resistance in Klebsiella Pneumoniae. J Glob Antimicrob Re. 2021;25:26-34.

15. Tsakris A, Poulou A, Pournaras S, Voulgari E, Vrioni G, Themeli-Digalaki K, Petropoulou D, Sofianou D. A Simple Phenotypic Method for the Differentiation of Metallo-Beta-Lactamases and Class a KPC Carbapenemases in Enterobacteriaceae Clinical Isolates. J. Antimicrob. Chemother. 2010;65(8):1664-71.

16. Qin X, Yang Y, Hu F, Zhu D. Hospital Clonal Dissemination of Enterobacter Aerogenes Producing Carbapenemase KPC-2 in a Chinese Teaching Hospital. J Med Microbiol. 2014; 63:222-28.

17. Poirel L, Walsh TR, Cuvillier V, Nordmann P. Multiplex PCR for Detection of Acquired Carbapenemase Genes. Diagn Micr Infec Dis. 2011;70(1):119-23.

18. Shon AS, Bajwa RP, Russo TA. Hypervirulent (Hypermucoviscous) Klebsiella Pneumoniae: A New and Dangerous Breed. Virulence. 2013;4(2):107-18.

19. Nadasy KA, Domiati-Saad R, Tribble MA. Invasive Klebsiella Pneumoniae Syndrome in North America. Clin Infect Dis. 2007;45(3): e25-28.

20. Yu F, Lv J, Niu S, Du H, Tang YW, Pitout JDD, Bonomo RA, Kreiswirth BN, Chen L. Multiplex PCR Analysis for Rapid Detection of Klebsiella pneumoniae Carbapenem-Resistant (Sequence Type 258 [ST258] and ST11) and Hypervirulent (ST23, ST65, ST86, and ST375) Strains. J Clin Microbiol. 2018;56(9) : e00776-18.

21. Fang CT, Chuang YP, Shun CT, Chang SC, Wang JT. A Novel Virulence Gene in Klebsiella Pneumoniae Strains Causing Primary Liver Abscess and Septic Metastatic Complications. J Exp Med. 2004;199(5):697705.

22. Turton JF, Baklan H, Siu LK, Kaufmann ME, Pitt TL. Evaluation of a Multiplex PCR for Detection of Serotypes K1, K2 and K5 in Klebsiella Sp. And Comparison of Isolates within these Serotypes. Fems Microbiol Lett. 2008;284(2):247-52.

23. Fang CT, Lai SY, Yi WC, Hsueh PR, Liu KL, Chang SC. Klebsiella Pneumoniae Genotype K1: An Emerging Pathogen that Causes Septic Ocular Or Central Nervous System Complications From Pyogenic Liver Abscess. Clin Infect Dis .2007;45(3):284-93.

24. Liu YC, Cheng DL, Lin CL. Klebsiella Pneumoniae Liver Abscess Associated with Septic Endophthalmitis. Arc Intern Med. 1986;146(10):1913-16.

25. Ning JZ. National Bureau of Statistics of China. Main Data of the Seventh National Population Census News Release. 2021. http://www.stats.gov.cn/ Main Data of the Seventh National Population Census News Release. Accessed 11 May 2021.

26. Ye M, Tu J, Jiang J, Bi Y, You W, Zhang Y, Ren J, Zhu T, Cao Z, Yu Z, Shao C, Shen Z, Ding B, Yuan J, Zhao X, Guo Q, Xu X, Huang J, Wang M. Klebsiella pneumoniaeClinical and Genomic Analysis of Liver AbscessCausing Identifies New Liver Abscess-Associated Virulence Genes. Front Cell Infect Mi. 2016;6:165.

27. Rossi B, Gasperini ML, Leflon-Guibout V, Gioanni A, de Lastours V, Rossi G, Dokmak S, Ronot M, Roux O, Nicolas-Chanoine MH, Fantin B, Lefort A. Hypervirulent Klebsiella Pneumoniae in Cryptogenic Liver Abscesses, Paris, France. Emerg Infect Dis. 2018;24(2):221-29.

28. Russo TA, Marr CM. Hypervirulent Klebsiella Pneumoniae. Clin Microbiol Rev. 2019;32(3) :e00001-19.

29. Zhang R, Liu L, Zhou H, Chan EW, Li J, Fang Y, Li Y, Liao K, Chen S. Nationwide Surveillance of Clinical Carbapenem-Resistant Enterobacteriaceae (CRE) Strains in China. Ebiomedicine. 2017;19: 98-106. 
30. Hao M, Shi X, Lv J, Niu S, Cheng S, Du H, Yu F, Tang YW, Kreiswirth BN, Zhang H, Chen L. In vitro Activity of Apramycin Against Carbapenem-Resistant and Hypervirulent Isolates. Front Microbiol. 2020;11: 425.

31. Shirley M. Ceftazidime-Avibactam: A Review in the Treatment of Serious Gram-Negative Bacterial Infections. Drugs. 2018;78(6):675-92.

32. Ehmann DE, Jahić H, Ross PL, Gu RF, Hu J, Kern G, Walkup GK, Fisher SL. Avibactam is a Covalent, Reversible, Non-B-Lactam B-Lactamase Inhibitor. P Natl Acad Sci Usa. 2012;109(29):11663-68.

33. Zhang P, Shi Q, Hu H, Hong B, Wu X, Du X, Akova M, Yu Y. Emergence of Ceftazidime/Avibactam Resistance in Carbapenem-Resistant Klebsiella Pneumoniae in China. Clinical microbiology and infection : the official publication of the European Society of Clinical Microbiology and Infectious Diseases. 2020;26(1):121-24.

34. Cui X, Shan B, Zhang X, Qu F, Jia W, Huang B, Yu H, Tang YW, Chen L, Du H. Klebsiella pneumoniae Reduced Ceftazidime-Avibactam Susceptibility in KPC-Producing From Patients without CeftazidimeAvibactam Use History - a Multicenter Study in China. Front Microbiol. 2020; 11:1365.

35. Bulger J, MacDonald U, Olson R, Beanan J, Russo TA. Metabolite Transporter PEG344 is Required for Full Virulence of Hypervirulent Klebsiella pneumoniae Strain hvKP1 after Pulmonary but Not Subcutaneous Challenge. Infect Immun. 2017;85(10)

36. Yonekawa S, Mizuno T, Nakano R, Nakano A, Suzuki Y, Asada T, Ishii A, Kakuta N, Tsubaki K, Mizuno S, Ogawa M, Yano H, Kasahara K, Mikasa K. Molecular and Epidemiological Characteristics of Carbapenemase-Producing Klebsiella pneumoniae Clinical Isolates in Japan. Msphere. 2020;5(5) :e0049020.

37. Yang Q, Jia X, Zhou M, Zhang H, Yang W, Kudinha T, Xu Y. Klebsiella pneumoniae Emergence of ST11-K47 and ST11-K64 Hypervirulent Carbapenem-Resistant in Bacterial Liver Abscesses From China: A Molecular, Biological, and Epidemiological Study. Emerg Microbes Infec. 2020;9(1):320-31.

38. Liao W, Liu Y, Zhang W. Virulence Evolution, Molecular Mechanisms of Resistance and Prevalence of ST11 Carbapenem-Resistant Klebsiella Pneumoniae in China: A Review Over the Last 10 Years. J Glob Antimicrob Re. 2020;23: 174-80.

39. Lee CR, Lee JH, Park KS, Kim YB, Jeong BC, Lee SH. Global Dissemination of Carbapenemase-Producing Klebsiella pneumoniae: Epidemiology, Genetic Context, Treatment Options, and Detection Methods. Front Microbiol. 2016; 7:895.

40. Lan P, Jiang Y, Zhou J, Yu Y. A Global Perspective On the Convergence of Hypervirulence and Carbapenem Resistance in Klebsiella Pneumoniae. J Glob Antimicrob Re. 2021; 25:26-34.

41. Zhou K, Xiao T, David S, Wang Q, Zhou Y, Guo L, Aanensen D, Holt KE, Thomson NR, Grundmann H, Shen P, Xiao Y. Novel Subclone of Carbapenem-Resistant Klebsiella Pneumoniae Sequence Type 11 with Enhanced Virulence and Transmissibility, China. Emerg Infect Dis. 2020;26(2):289-97.

42. Zhang X, Ouyang J, He W, Zeng T, Liu B, Jiang H, Zhang Y, Zhou L, Zhou H, Liu Z, Liu L. Co-Occurrence of Rapid Gene Gain and Loss in an Interhospital Outbreak of Carbapenem-Resistant Hypervirulent ST11-K64 Klebsiella pneumoniae. Front Microbiol. 2020; 11:2518.

43. Rodríguez-Baño J, Gutiérrez-Gutiérrez B, Machuca I, Pascual A. Treatment of Infections Caused by Extended-Spectrum-Beta-Lactamase-, AmpC-, and Carbapenemase-Producing Enterobacteriaceae. Clin Microbiol Rev. 2018;31(2): e00079-17. 
44. Li L, Yuan Z, Chen D, Xie X, Zhang B. Klebsiella pneumoniae Clinical and Microbiological Characteristics of Invasive and Hypervirulent Infections in a Teaching Hospital in China. Infect Drug Resist. 2020; 13:4395403.

45. Zheng X, Wang JF, Xu WL, Xu J, Hu J. Klebsiella pneumoniaeClinical and Molecular Characteristics, Risk Factors and Outcomes of Carbapenem-resistant Bloodstream Infections in the Intensive Care Unit. Antimicrob Resist in. 2017; 6:102.

46. Jiao Y, Qin Y, Liu J, Li Q, Dong Y, Shang Y, Huang Y, Liu R. Risk Factors for Carbapenem-Resistant Klebsiella Pneumoniae Infection/Colonization and Predictors of Mortality: A Retrospective Study. Pathog Glob Health. 2015;109(2):68-74.

\section{Tables}

Table1. Microbiological and clinical characteristics of CR-hvKP strains 
Characteristic

CR-

hvKP(N=51)

CR-non-

hvKP(N=29)

Microbiological characteristics

\section{Capsular serotype}

\begin{tabular}{|c|c|c|c|}
\hline k64 & $44 \rrbracket 86.3 \rrbracket$ & $18 \otimes 62.1 \rrbracket$ & 0.013 \\
\hline k47 & $1 \otimes 2.0 \bigotimes$ & 1囚3.4囚 & 0.684 \\
\hline K54 & $1 \otimes 2.0 \rrbracket$ & $0 \otimes 0.0 \otimes$ & 0.451 \\
\hline K-non-typable & $5 \otimes 9.8 \rrbracket$ & $10 \rrbracket 34.5 \rrbracket$ & 0.007 \\
\hline \multicolumn{4}{|l|}{ MLST } \\
\hline ST11 & $45 \rrbracket 88.2)$ & $20 \otimes 69.0 \otimes$ & 0.034 \\
\hline ST15 & $5(9.8)$ & $8(27.6)$ & 0.039 \\
\hline ST198 & $1 \otimes 2.0 \otimes$ & $0 \rrbracket 0.0 \rrbracket$ & 0.451 \\
\hline ST438 & $0 \rrbracket 0.0 \rrbracket$ & $1 \rrbracket 3.4 \rrbracket$ & 0.185 \\
\hline
\end{tabular}

\section{ST-capsular serotype}

ST11-K64

$44 \llbracket 86.3 \rrbracket$

17(58.6)

0.005

ST11-K47

1(2.0)

1(3.4)

0.684

ST11-K-non-typable

$0(0.0)$

2(6.9)

0.256

ST15-K54

$1(2.0)$

0

0.451

ST15- K-non-typable

4(7.8)

$8(27.6)$

0.017

ST198- K-non-typable

$1(2.0)$

0

0.451

ST438-K64

0

1(3.4)

0.185

\section{Basic demographics}

Age『median

85.0

$\bigotimes 75.0 \sim 88.0 \rrbracket$

Male

Underlying diseases

Pulmonary disease

diabetes mellitus

Hypertension

Cerebrovascular Diseases

Cardiovascular disease

Malignancy

$\begin{array}{lll}40 \otimes 78.4 \rrbracket & 20(69.0 \rrbracket & 0.347\end{array}$

47(92.2)

28(96.6)

0.435

26(51.0)

16ख55.2)

0.718

30(58.8)

17(58.6)

0.986

25(49.0)

16(55.2)

0.597

$31(60.8)$

$5(17.2)$

$<0.001$

$4(7.8)$

7(24.1)

0.087 
Hypoproteinemia

10(19.6)

6(20.7)

0.907

liver abscess

$4(7.8)$

$1 \rrbracket 3.4 \rrbracket$

0.648

\section{Invasive procedures and devices}

Surgery within 1 month

4\7.8区

$6 \rrbracket 20.7 \rrbracket$

0.097

Mechanical ventilation

$41 \rrbracket 80.4 \rrbracket$

$23 \rrbracket 79.3 \rrbracket$

0.907

Central venous catheter

$35 \rrbracket 68.6 \rrbracket$

$25 \rrbracket 86.2 \rrbracket$

0.081

Urinary catheter

42\82.4囚

$27 \rrbracket 93.1 \rrbracket$

0.907

Gastric tube

41邓80.4邓

$28 \llbracket 96.6 \rrbracket$

0.045

\section{Antibiotic exposure}

Cephamycins

7(13.7)

5(17.2)

0.674

Penicillin

7(13.7)

6(20.7)

0.420

Cephalosporins

27(52.9)

$16(55.2)$

0.847

$\beta$-lactam/ $\beta$-lactamase inhibitors

33(64.7)

17(58.6)

0.589

Fluoroquinolones

28(54.9)

18(62.1)

0.533

Carbapenems

33(64.7)

22(75.9)

0.301

Tigecycline

27(52.9)

13(44.8)

0.485

Polymyxin B

$3 \llbracket 5.9 \rrbracket$

1区3.4区

0.633

Glycopeptides

12(23.5)

2(6.9)

0.06

Aminoglycosides

6(11.8)

4(13.8)

0.793

Fosfomycin

$4(7.8)$

3(10.3)

0.705

Hospitalization within last 90 days

22(43.1)

7(24.1)

0.089

\section{Outcomes}

Clinical improvement without modification of initial treatment

Change of initial antibiotics due to clinical worsening

Bacterial clearance after $72 \mathrm{~h}$ treatment

Persistent infection after $72 \mathrm{~h}$ treatment

stable,discharged

In-hospital mortality

length of stay $[$ median

$\begin{array}{lll}8(15.7) & 4(13.8) & 0.821\end{array}$

$30(58.8)$

10(34.5)

0.036

2(3.9)

2(6.9)

0.56

$40(78.4)$

17(58.6)

0.06

25(49.0)

14(48.3)

0.949

21(41.2)

15(51.7)

0.362

24ه15 47ه

22(16.5 34.5》

0.417 
Table 2. Susceptibility of CR-hvKP and CR-non-hvKP strains against different antimicrobial agents

\begin{tabular}{|c|c|c|c|c|c|c|c|}
\hline \multirow{2}{*}{ Antibiotic agents } & \multicolumn{3}{|c|}{ CR-hvKP $(n=51)$} & \multicolumn{4}{|c|}{ CR-non-hvKP(n=29) } \\
\hline & $\begin{array}{l}\text { MIC50 } \\
(\mu \mathrm{g} / \mathrm{mL})\end{array}$ & $\begin{array}{l}\text { MIC90 } \\
(\mu \mathrm{g} / \mathrm{mL})\end{array}$ & $\% \mathrm{R}$ & $\begin{array}{l}\text { MIC50 } \\
(\mu \mathrm{g} / \mathrm{mL})\end{array}$ & $\begin{array}{l}\text { MIC90 } \\
(\mu \mathrm{g} / \mathrm{mL})\end{array}$ & $\% R$ & $\begin{array}{l}\mathrm{P} \\
\text { Value }\end{array}$ \\
\hline Ceftazidime & $>128$ & $>128$ & $100.0 \%$ & 128 & $>128$ & $100.0 \%$ & I \\
\hline Ceftazidime/avibactam & $4 / 4$ & 8 & $2.0 \%$ & $2 / 4$ & 8 & $3.5 \%$ & 0.684 \\
\hline Cefepime & $>128$ & $>128$ & $96.1 \%$ & $>128$ & $>128$ & $100.0 \%$ & 0.283 \\
\hline Aztreonam & $>128$ & $>128$ & $100.0 \%$ & $>128$ & $>128$ & $100.0 \%$ & l \\
\hline Piperacillin/Tazobactam & $>512 / 4$ & $>512 / 4$ & $100.0 \%$ & $>512 / 4$ & $>512 / 4$ & $100.0 \%$ & I \\
\hline Imipenem & $>32$ & $>32$ & $100.0 \%$ & $>32$ & $>32$ & $100.0 \%$ & / \\
\hline Meropenem & $>32$ & $>32$ & $98.0 \%$ & $>32$ & $>32$ & $100.0 \%$ & 0.451 \\
\hline Imipenem/avibactam a & $0.5 / 4$ & $2 / 4$ & $3.9 \%$ & $0.25 / 4$ & $1 / 4$ & $3.5 \%$ & 0.915 \\
\hline Amikacin & $>512$ & $>512$ & $86.3 \%$ & $>512$ & $>512$ & $58.6 \%$ & 0.050 \\
\hline Polymyxin $B^{b}$ & 0.5 & 1 & $0.0 \%$ & 0.5 & 16 & $17.2 \%$ & 0.002 \\
\hline Tigecycline & 2 & 2 & $0.0 \%$ & 1 & 2 & $0.0 \%$ & / \\
\hline Levofloxacin & 64 & $>64$ & $100.0 \%$ & 32 & 64 & $100.0 \%$ & I \\
\hline $\begin{array}{l}\text { Cefoperazone- } \\
\text { Sulbactam }^{a}\end{array}$ & $256 / 128$ & $>256 / 128$ & $100.0 \%$ & $>256 / 128$ & $>256 / 128$ & $100.0 \%$ & / \\
\hline
\end{tabular}

a: Breakpoints for imipenem/avibactam and cefoperazone/sulbactam have not yet been determined, therefore breakpoints for imipenem and cefoperazone have been applied.

b: Colistin MIC results can predict the sensitivity of polymyxin B, therefore breakpoints for Colistin have been applied.

Table 3. Univariate and Multivariate Logistic Regression Analysis of CR-hvKP infections

\begin{tabular}{lllll} 
Variables & \multicolumn{2}{c}{ Univariate Analysis } & & Multivariable Analysis \\
\cline { 2 - 5 } & OR $(95 \% \mathrm{Cl})$ & P value & OR $(95 \% \mathrm{Cl})$ & P value \\
\hline ST11-K64 & $4.437(1.496-13.161)$ & 0.005 & $8.385 \rrbracket 2.017-34.858 \rrbracket$ & 0.003 \\
\hline Cardiovascular disease & $7.440 \rrbracket 2.438-22.700 \rrbracket$ & $<0.001$ & $11.956 \rrbracket 3.072-46.538 \rrbracket$ & $<0.001$
\end{tabular}

\section{Figures}




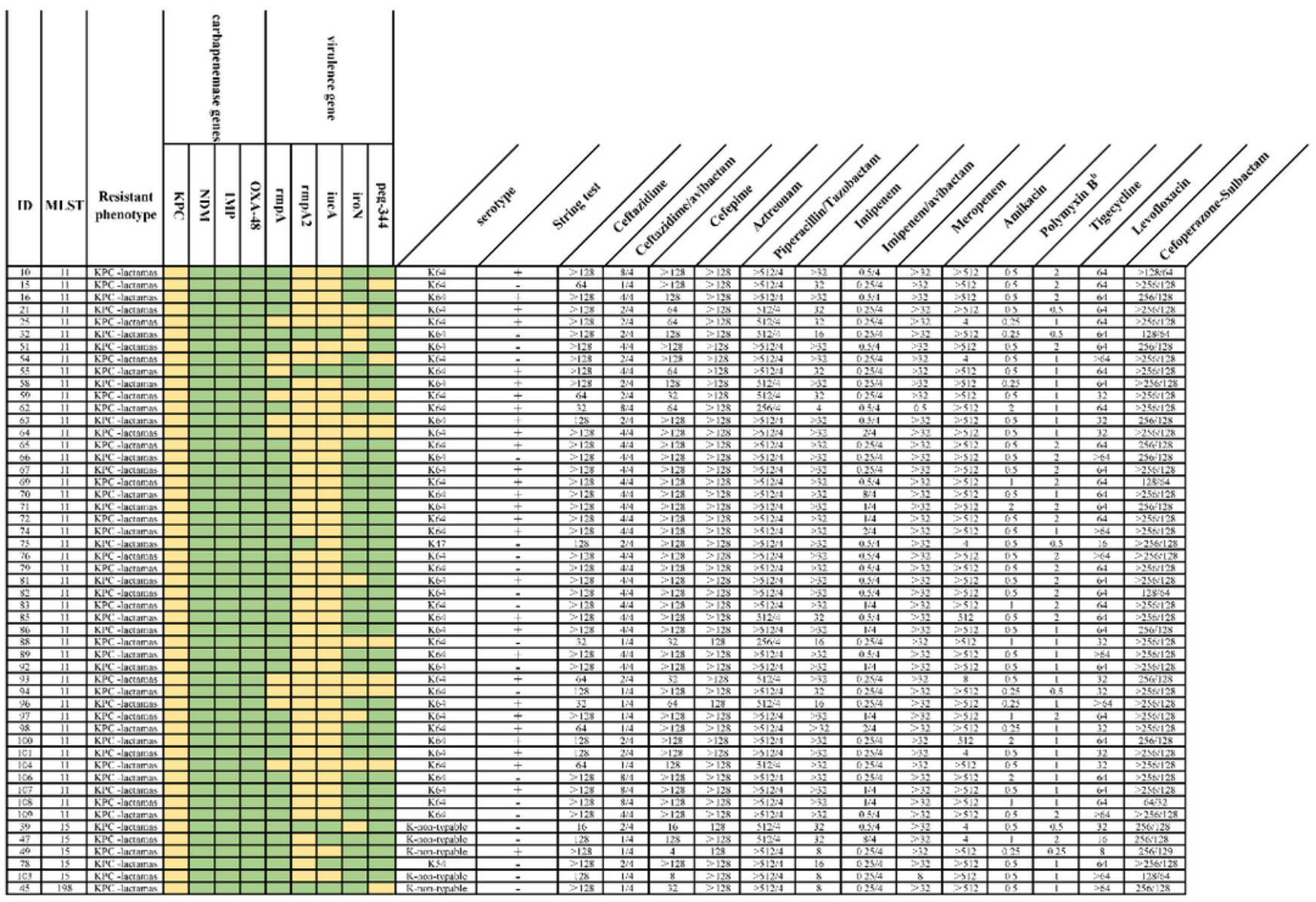

\section{Figure 1}

MLST, resistance and virulence factors, and MIC values of 51 CR-hvKP strains. MLST, resistance and virulence factors and MICs are shown on the left, middle and right, respectively. The presence of genes in a specific genome is represented by the light yellow box and the absence of genes is represented by a green box. Each row of the heatmap (middle) indicates a strain, and each column represents a gene that belongs to the indicated functional clusters shown at the top.

\section{Supplementary Files}

This is a list of supplementary files associated with this preprint. Click to download.

- Primers6.xlsx 